\title{
Variación estacional de aves en el humedal de Trumao, centro-sur de Chile
}

\author{
Claudio N. Tobar' (1), Diego Meier' ${ }^{1}$ Jaime R. Rau² (D), Catalina Ríos-Henríquez³ (D), \\ Héctor Pavés ${ }^{1,4}$ (i) \& Alexis Santibáñez
}

\footnotetext{
1. Departamento de Ciencias Básicas, Facultad de Ciencias, Universidad Santo Tomás, Los Carrera 753, Osorno, Chile. (claudiotobarbo@santotomas.cl; hectorpaveshe@santotomas.cl; alexissantibanez@santotomas.cl)

2. Laboratorio de Ecología, Depto. de Ciencias Biológicas \& Biodiversidad \& Programa IBAM, Universidad de Los Lagos, Casilla 933, Campus Osorno, Osorno, Chile. (jrau@ulagos.cl)

3. Laboratorio de Limnología, Departamento de Acuicultura \& Recursos Agroalimentarios, Universidad de Los Lagos, Campus Osorno, Casilla 933, Osorno, Chile. (catalina.rios@ulagos.cl)

4. Centro de Investigación e Innovación para el Cambio Climático (CiiCC), Facultad de Ciencias, Universidad Santo Tomás, Santiago, Chile.
}

ABSTRACT. Seasonal variation of birds in Trumao wetland, south-central Chile. This study describes the assemblage and habitat use of birds in the Trumao wetland and its seasonal variation according to the hydrological regime during spring 2017 and summer 2018 (not flooded) and winters 2017 and 2018 (flooded). Overall, 29 species of birds were recorded with a total abundance of 2,341 individuals. The total number of accumulated species observed for the flooded and non-flooded period corresponded to 16 and 22 species, respectively, with the Brown hooded Gull [Chroicocephalus maculipennis (Lichtenstein, 1823)] being the dominant species. The qualitative Sorensen similarity index between the flooding and non-flooding periods of the wetland was $73.2 \%$, while the quantitative Bray-Curtis similarity was $32.65 \%$. When comparing the flooded winter period (4 m depth) and the non-flooded summer period (1 m depth), the diversity presented statistically significant differences, but not the abundance. The SIMPER analysis showed that between the flooded winter period and the non-flooded summer period, the species that showed a greater percentage of dissimilarity in their abundance between these seasons were the Brown hooded Gull and the Slender-billed Parakeet [Enicognathus leptorhynchus (King,1831)]. The use of habitat was highly variable, both seasonally and spatially. Our results indicate that birds occupy wetlands according to seasonal fluctuations and according to the habitat that is available; however, abundance is not affected by hydrological changes. It is essential to continue carrying out more research in the wetland to determine the nesting, feeding and reproduction of the birds in the different habitats used to generate its protection and conservation measures in the Trumao wetland.

KEYWORDS. Aquatic birds, conservation, habitat, Chroicocephalus maculipennis.

RESUMEN. En este estudio se describe el ensamble de aves del humedal de Trumao y su variación estacional según régimen hidrológico durante primavera 2017 y verano 2018 (no inundado) e inviernos 2017 y 2018 (inundado) y se evalúa el tipo de hábitat. Se registraron 29 especies de aves con una abundancia total de 2,341 individuos. El número total de especies acumuladas observadas para el período inundado y no inundado correspondió a 16 y 22 especies, respectivamente, siendo Chroicocephalus maculipennis (Lichtenstein, 1823) (Gaviota cáhuil) la especie dominante durante el período de estudio. El índice de similitud de Sorensen entre los períodos de inundación y no inundación del humedal fue de $73.2 \%$, mientras que la similitud de Bray-Curtis resultó en un 32.65\%. Al compararse el periodo invernal inundado (4 m profundidad) y el periodo estival no inundado ( $1 \mathrm{~m}$ profundidad), el índice de diversidad registró diferencias estadísticamente significativas, en cambio, la abundancia no presentó diferencias significativas. El análisis SIMPER arrojó que entre el periodo invernal inundado y el periodo estival no inundado las especies que mostraron un mayor porcentaje de disimilitud en su abundancia entre estas temporadas fueron la Gaviota cáhuil y Loro choroy [Enicognathus leptorhynchus (King, 1831)]. El uso de hábitat en el humedal de Trumao, por parte de las aves, fue altamente variable, tanto estacional como espacialmente, resultado esperable para este tipo de ecosistemas. Finalmente, nuestros resultados nos indican que las aves ocupan los humedales según fluctuaciones estacionales y según el hábitat que se encuentre disponible, sin embargo, la abundancia no se ve afectada por los cambios hidrológicos. Con estos resultados es fundamental seguir realizando más investigaciones en el humedal para determinar el uso (nidificación, alimentación, reproducción) que realizan las aves en los distintos hábitats para de esta manera generar medidas de protección y conservación para la gestión del humedal de Trumao.

PALABRAS CLAVE. Aves acuáticas, conservación, hábitat, Chroicocephalus maculipennis.

Los humedales se encuentran entre los ecosistemas más productivos del planeta, brindado sustento alimenticio, estético y cultural a varias civilizaciones del mundo (MONTOYA, 2000), su funcionamiento es altamente dinámico y albergan una rica diversidad de flora y fauna (CANTERO, 1993). Dentro de esta gran diversidad, las aves habitan los humedales a lo largo de su ciclo anual, como sitios de anidación y forrajeo, transformándose en áreas importantes de concentración, especialmente para las aves migratorias (GAUTHIER et al., 2005; BILDSTEIN et al., 1991), cumpliendo un rol ecológico dentro de estos ambientes, pueden oxigenar el ambiente bentónico mientras se alimentan y aportar nutrientes a la columna de agua por medio de las heces y desperdicios de comida (ONUF et al., 1977; NAVEDo et al., 2015). Tanto su 
riqueza como su abundancia dependen de diversos factores, como el régimen hidrológico, la estructura de la vegetación y la heterogeneidad ambiental (WeLLER, 1999; POHORYLOW et al., 2016). Uno de los factores importantes en los humedales es el régimen hidrológico porque provoca cambios en la estructura del hábitat y disponibilidad de alimento, incidiendo en la variación temporal de la riqueza y diversidad de aves (KNUTSON \& KLASS, 1997; KINGSFORD et al., 2004; CUMMING et al., 2012; LoRENZÓN et al., 2017, 2019). Estos cambios en el régimen hidrológico están dados por la estacionalidad climática, es decir, temporadas inundadas en otoño-invierno y no inundadas en primavera-verano forzando la variabilidad temporal tanto en aves residentes como en migrantes (LORENZÓN et al., 2019), es decir, utilizan los humedales de manera oportunista, ya que cuando las condiciones no son las más óptimas (inundaciones/sequías), abandonan el ecosistema porque el alimento se vuelve inaccesible, además estás fluctuaciones impulsan patrones de anidamiento temporal, provocando cambios en el éxito reproductivo, tornándose más escaso (KNUTSON \& KLASs, 1997), además, las variaciones del régimen hidrológico, fuerza a que en el ecosistema exista una combinación de diferentes tipos de hábitat y las aves los utilizan según los requerimientos de cada especie (LoRENZÓN et al., 2019). La respuesta del ensamble de aves con respecto a los cambios en el régimen hidrológico ha sido escasamente estudiada, cuando disminuye el espejo de agua aumentan las aves terrestres, viéndose afectadas el grupo de aves que se alimentan desde aguas profundas y lo contrario ocurre cuando aumenta la columna de agua, aumenta la población de aves acuáticas, disminuyendo la presencia de aves terrestres (por ejemplo, KNUTSON \& KLASS, 1997; KInGSFORD et al., 2004; CUMMING et al., 2012; BODMER et al., 2018; LoRENZÓN et al., 2019).

En Chile existen más de 40,000 humedales (WCS, 2019), sin embargo, solo 13 de ellos presentan medidas de conservación (MMA, 2018). La baja protección es debida a la escasa información sobre la estructura biótica de los humedales y de su funcionamiento integral (GALLARDO et al., 2018), siendo necesario ampliar el conocimiento faunístico en los humedales, siendo una herramienta fundamental para su conservación y gestión (ACOSTA \& MURÚA, 2002). Hoy en día, existen muchos humedales que no presentan medidas de protección ni de conservación, como es el caso del humedal de Trumao. Este humedal es un área de importancia para las aves acuáticas, especialmente anátidas (patos silvestres) (SAG, 2015), se encuentra fuertemente amenazado por la expansión urbana, caza ilegal de aves, pesca furtiva, presencia de especies invasoras como el Neovison vison Schreber, 1777 (visón) y la contaminación por parte de la industria agropecuaria y acuícola, sumado al hecho del escaso conocimiento de las aves acuáticas que habitan en estos humedales y su estado de conservación. La ejecución de programas de conservación de fauna implica la prospección y evaluación de toda la riqueza faunística a proteger (MARTORI et al., 2002). Por lo anterior, los objetivos de este estudio fueron describir el ensamble de aves del humedal de Trumao, centro-sur de Chile y determinar su variación estacional durante periodos secos estivales (primavera 2017 y verano 2018) e inundados invernales (inviernos 2017 y 2018), además, evaluar el uso de hábitat de las especies registradas durante las fluctuaciones hidrológicas del humedal.

\section{MATERIALES Y MÉTODOS}

Área de estudio. El humedal de Trumao (40²1'1.46”'S - 73746.33”O) se encuentra ubicado en la cuenca del Río Bueno entre la Región de Los Ríos y Los Lagos, (Fig.1); es la quinta con mayor caudal de Chile (DGA, 2004). A $65 \mathrm{~km}$ de la desembocadura se unen tres grandes ríos para continuar en uno solo, formándose un humedal fluvial de unas 8,325 hectáreas de fondo de valle, unos $25 \mathrm{~km}$ de largo y $2 \mathrm{~km}$ de ancho, a escasos metros sobre el nivel del mar (DGA, 2004). Con una dinámica hídrica compleja de rebalses invernales en el fondo de valle y una marea diaria de hasta un metro, presentando una influencia diaria de mareas oceánicas con variables similares de estuario, aunque durante pleamar no existe escurrimiento hacia la desembocadura, sino en sentido contrario. A esta dinámica diaria, se suma una dinámica anual de inundaciones y no inundaciones del humedal, provocada por la variación estacional por los aumentos del caudal en invierno debido a las precipitaciones $(1,800 \mathrm{~mm}$ anuales promedio y $75 \%$ del total entre mayo y septiembre), generando desbordes del cauce e inundaciones de al menos el 48\% (4,000 ha) del humedal, creando grandes lagunas. A partir de octubre, época de primavera-verano, comienza una marcada baja del cauce por la disminución de las precipitaciones, entonces las grandes lagunas invernales desaparecen, transformándose al menos el $65 \%$ en praderas de gramíneas y solo un $35 \%$ se mantiene como pequeños esteros anegados en la época seca estival (DGA, 2004). A partir de la particular dinámica de inundaciones invernales del $48 \%$ de la superficie del humedal y su posterior transformación en pastizales en verano, se generan dos ambientes con condiciones de hábitat marcadamente diferentes con una marcada variación temporal.

Metodología de muestreo. Durante las estaciones de invierno 2017 (julio a septiembre), primavera 2017 (octubre a diciembre), verano 2018 (enero a marzo) e invierno 2018 (julio a septiembre) se realizaron observaciones y conteos de las aves presentes en el humedal de Trumao, mediante puntos fijos de conteo por un tiempo de 10 minutos (BIBBy et al., 2000), por dos días seguidos entre las 8:00 am a 12:00 am, empleando lugares en altura o refugios mimetizados con el entorno para que la observación no afecte la normal conducta de la fauna. Se establecieron cuatro puntos de observación denominados: 1) Pto. Trumao La Unión, 2) Pto. Trumao Quilacahuin, 3) Isla Momberg, 4) Currupulli (Fig. 1) los cuales estaban a una distancia de aproximadamente $10 \mathrm{~km}$, los cuales fueron seleccionados por la presencia de espejos de agua durante la época invernal. Todos los registros se realizaron con un binocular (Bushnell Trophy XLT) (8X30) y telescopio terrestre (Barska Naturescape) (60X). La riqueza y abundancia de aves fueron agrupadas según las estaciones del año: verano (enero a marzo), invierno (julio 

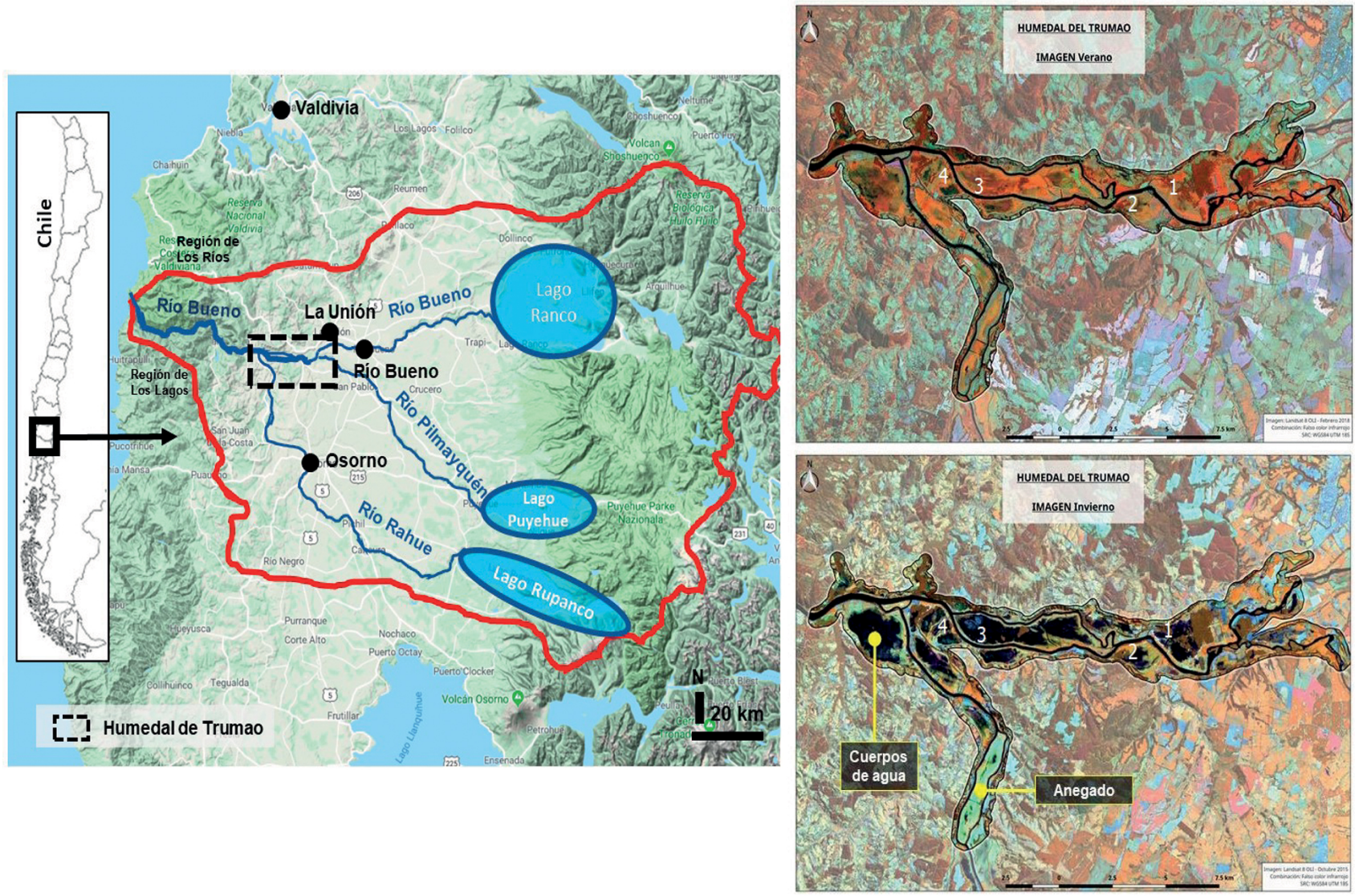

Fig. 1. Área de estudio, cuenca del Río Bueno, Chile, y ubicación del humedal de Trumao (rectángulo negro). Humedal de Trumao durante la estación de primavera 2017 y verano 2018 (no inundado) e inviernos 2017 y 2018 (inundado). Destacan los puntos de monitoreo de aves: 1, Pto. Trumao La Unión; 2, Pto. Trumao Quilacahuin; 3, Isla Momberg; 4, Currupulli.

a septiembre) y primavera (octubre a diciembre), la época de otoño no fue incluida debido a que solo se consideraron los periodos representativos de las épocas inundadas $(4 \mathrm{~m}$ profundidad) y no inundadas (1 $\mathrm{m}$ profundidad) (DGA, 2004). La abundancia estacional correspondió al promedio de aves registradas en cada conteo para cada estación anual (CuRsaCh et al., 2010), mientras que la riqueza estacional correspondió al número total de especies registradas en los conteos agrupadas por estación climática. El uso de hábitat se determinó basándose en los requerimientos de microhábitat por observación directa, complementado con lo reportado en la literatura (COUVE \& VIDAL, 2003; BARROS et al., 2015). De esta manera, las aves se clasificaron en dos grandes grupos: (1) acuáticas y (2) terrestres de pastizales; el primer grupo requiere total o parcialmente de ambientes húmedos (asociados a cuerpos o cursos de agua) en sus ciclos de vida (Couve \& VIDAL, 2003) y el segundo grupo son aves que tienen un ciclo de vida terrestre, pero que utilizan ambientes húmedos o adyacentes a estos de manera oportunista (FIGUEROA et al., 2000).

Análisis estadístico. Para efectos de comparación estadística, se utilizaron las estaciones climáticas con inundación (inviernos) y no inundación (primavera 2017 y verano 2018). La diversidad de aves se obtuvo utilizando el índice de Shannon-Wiener $\left(\log 10 \mathrm{H}^{\prime}\right)$, mediante el cual se obtienen parámetros completos de la diversidad de especies cuantificando en una sola cifra el número de especies y su representatividad en un hábitat (MoRENo, 2001), el cual se obtuvo mediante el uso del programa Biodiversity Professional Beta 1 (McAleEce, 1997). Para estimar diferencias significativas de diversidad (H'), entre las estaciones de invierno 2017, primavera 2017, verano 2018 e invierno $2018(P<0,05)$ para cada punto de monitoreo, se utilizó la prueba de Hutcheson (véase Moreno, 2001), la cual es análoga a la prueba t de Student y permite comparar dos índices de diversidad y sus varianzas tanto espacial como temporalmente (ZAR, 1999). Se obtuvo el índice de similitud de Bray-Curtis, para comparar el total de especies acumuladas entre los periodos de inundación y de sequía del humedal, además, se realizó un análisis de conglomerados para determinar el grado de similitud avifaunística entre los distintos puntos de monitoreo para las distintas estaciones climáticas, para lo anterior se utilizó el programa Biodiversity Professional Beta 1 (McAleEce, 1997). Para comparar la similitud del número de especies acumuladas entre los periodos de inundación y no inundación del humedal se utilizó el coeficiente de similitud de Sorensen (Moreno, 2001). Para obtener el porcentaje de disimilitud cualitativa de 
las especies entre y dentro del periodo invernal inundado y del periodo estival no inundado se procedió a realizar un análisis SIMPER y para observar el uso de hábitat en ambos períodos se realizó un Análisis de Escalamiento Multidimensional no Métrico (MDS por sus siglas en inglés), para ambos análisis se utilizó el software PRIMER 6.6.14 (ClaRKE \& Gorley, 2006). Puesto que los datos de conteos no siguen distribuciones normales, para comparar las medianas entre las abundancias de aves en humedales inundados y no inundados se utilizó la prueba no paramétrica U de MannWhitney la cual es análoga a la prueba t de Student para dos muestras independientes (ZAR, 1999). En el caso de la media aritmética y desviación estándar del número acumulado de especies para comparar las riquezas de especies se utilizó la prueba estadística binomial (ZAR, 1999). Para ambos análisis se utilizó el paquete computacional estadístico en línea VassarStats: Website for Statistical Computation (CRichard Lowry 1998-2017; <http://vassarstats.net/>).

\section{RESULTADOS}

Caracterización del ensamble de aves en el humedal de Trumao. Se registró un total de 29 especies de aves con una abundancia total de 2,341 individuos (Tab. I), siendo las más abundantes Chroicocephalus maculipennis (Lichtenstein, 1823) (Gaviota cáhuil), Enicognathus leptorhynchus (King, 1831) (Choroy), Vanellus chilensis (Molina, 1782) (Queltehue), Anas georgica (Gmelin, 1789) (Pato jergón grande) y Fulica armillata (Vieillot, 1817) (Tagua común), especies con estado de conservación igual a preocupación menor (Tab. I). Las mayores abundancias se obtuvieron en la estación de invierno 2018 en Pto. Trumao La Unión con 372 individuos y Pto. Trumao Quilacahuin con 368 individuos, mientras que la riqueza fue mayor en invierno 2018 en Pto. Trumao Quilacahuin con 15 especies y en primavera 2017 en Isla Momberg y Pto. Trumao Quilacahuin con 14 especies.

Variación estacional y uso de habitat. El análisis de conglomerados mostró que las mayores similitudes se registraron entre las estaciones estivales de verano 2018 entre Pto. Trumao La Unión y Currupulli $(69,7 \%)$ y verano 2018 en Pto. Trumao Quilacahuin y primavera 2017 en Currupulli (65,2\%) (Fig. 2). El número total de especies registradas para el periodo inundado fue de 16 especies y durante el periodo no inundado fue de 22 especies, respectivamente, y la similitud de Sorensen fue de $73,2 \%$. De acuerdo con la prueba estadística binomial las medias aritméticas y desviación estándar para el período inundado fueron $18,98 \pm 2,26$ y para el periodo no inundado $21,84 \pm$ 3,49 , valores que difirieron estadísticamente $(\mathrm{t}=2,2$; g.l. $=25 ; \mathrm{p}=0,037)$. Por otra parte, la similitud de especies acumuladas, obtenida con el índice de Bray-Curtis entre los períodos de inundación y no inundación del humedal fueron $32,65 \%$. El análisis SIMPER arrojó que entre el periodo invernal inundado y el periodo estival no inundado la disimilitud promedio en la abundancia fueron $79,55 \%$. Las especies que mostraron un mayor porcentaje de disimilitud entre estas temporadas fueron Chroicocephalus maculipennis (Gaviota cáhuil) con un 21,87\%, seguido de Enicognathus leptorhynchus (Choroy) con un 10,40\% y Anas georgica (Pato jergón grande) con un $9,63 \%$. Además, durante el periodo estival no inundado, la similitud fue de $37,29 \%$, siendo Bubulcus ibis (Linnaeus, 1758) (Garza boyera) la especie con mayor porcentaje $(18,45 \%)$, seguida de Vanellus chilensis (Queltehue) (17,46\%) y Theristicus melanopis (Boddaert, 1783) (Bandurria) (16,12\%). Finalmente, durante el periodo invernal inundado, la similitud fue de $26,64 \%$, donde la especies que presentaron mayor porcentaje de contribución a la similitud fueron Chroicocephalus maculipennis (Gaviota cáhuil) (27,99\%), Vanellus chilensis (Queltehue) (23,19\%) y Anas georgica (Pato jergón grande) (17,07\%). Al comparar la abundancia de aves durante el periodo estival no inundado y el periodo invernal inundado no se observaron diferencias estadísticas significativas ( $\mathrm{U}=76,5 ; \mathrm{p}=0,1112)$.

La diversidad de aves fue mayor en la estación climática de primavera 2017 en Pto. Trumao Quilacahuin $\left(H^{\prime}=1,065\right)$ e Isla Momberg $\left(H^{\prime}=0,935\right)$ (Fig. 3). Al comparar la diversidad estacional para cada punto monitoreado, de las 24 comparaciones realizadas se obtuvieron diferencias significativas para 16 comparaciones (Tab. II). Del total de especies, registramos que el $58,62 \%$ del ensamble utiliza el ambiente acuático mientras que $20,69 \%$ el ambiente terrestre, así como el 20,69 \% utilizan ambos ambientes. En el MDS se observa que la mayoría de las especies de ambientes acuáticos se distribuyen cercano a la línea del período inundado, así como también se observa las especies que se encuentran en ambos períodos (Fig. 4).

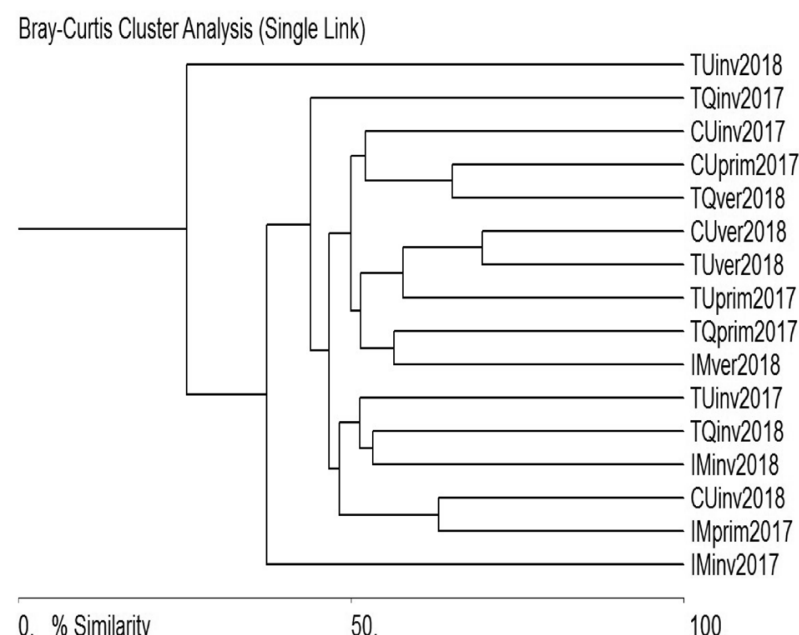

Fig. 2. Dendrograma de similitud para las abundancias de aves en las distintas estaciones climáticas en los distintos puntos de monitoreo. Las siglas corresponden a los siguientes sitios de monitoreo en Chile: IM, Isla Momberg; TU, Puerto Trumao La Unión; TQ, Puerto Trumao Quilacahuin y CU, Currupulli. A las siguientes temporadas: inv, invierno; prim, primavera; ver, verano; cada sigla está acompañada de su respectivo año de muestreo: 2017 y 2018. 


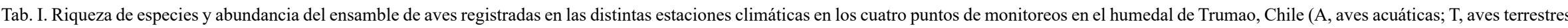
de pastizales; LC, Preocupación menor; EN, En peligro).

\begin{tabular}{|c|c|c|c|c|c|c|c|c|c|c|c|c|c|c|c|c|c|c|c|}
\hline \multirow[t]{2}{*}{ Especie } & \multirow[t]{2}{*}{$\begin{array}{l}\text { Nombre } \\
\text { común }\end{array}$} & \multirow{2}{*}{$\begin{array}{l}\text { Estado de } \\
\text { conserva- } \\
\text { ción }\end{array}$} & \multirow{2}{*}{$\begin{array}{c}\text { Uso } \\
\text { de } \\
\text { hábitat }\end{array}$} & \multicolumn{4}{|c|}{ Isla Momberg } & \multicolumn{4}{|c|}{ Pto. Trumao La Unión } & \multicolumn{4}{|c|}{ Pto. Trumao Quilacahuin } & \multicolumn{4}{|c|}{ Currupulli } \\
\hline & & & & $\begin{array}{l}\text { Inv } \\
2017\end{array}$ & $\begin{array}{l}\text { Prim } \\
2017\end{array}$ & $\begin{array}{l}\text { Ver } \\
2018\end{array}$ & $\begin{array}{c}\text { Inv } \\
2018\end{array}$ & $\begin{array}{l}\text { Inv } \\
2017\end{array}$ & $\begin{array}{l}\text { Prim } \\
2017\end{array}$ & $\begin{array}{c}\text { Ver } \\
2018\end{array}$ & $\begin{array}{c}\text { Inv } \\
2018\end{array}$ & $\begin{array}{l}\text { Inv } \\
2017\end{array}$ & $\begin{array}{l}\text { Prim } \\
2017\end{array}$ & $\begin{array}{c}\text { Ver } \\
2018\end{array}$ & $\begin{array}{c}\text { Inv } \\
2018\end{array}$ & $\begin{array}{c}\text { Inv } \\
2017\end{array}$ & $\begin{array}{l}\text { Prim } \\
2017\end{array}$ & $\begin{array}{c}\text { Ver } \\
2018\end{array}$ & $\begin{array}{c}\text { Inv } \\
2018\end{array}$ \\
\hline $\begin{array}{l}\text { Theristicus } \\
\text { melanopis }\end{array}$ & Bandurria & $\mathrm{LC}$ & $\mathrm{A} / \mathrm{T}$ & $22 \pm 13,5$ & $9 \pm 7,5$ & $8 \pm 4,5$ & $25 \pm 11,7$ & 6 & 6 & 6 & 7 & 61 & $3 \pm 1,2$ & $4 \pm 3,5$ & $8 \pm 3,5$ & 5 & 3 & $7 \pm 3,5$ & $12 \pm 0,7$ \\
\hline $\begin{array}{l}\text { Patagioenas } \\
\text { araucana }\end{array}$ & Torcaza & $\mathrm{LC}$ & $\mathrm{T}$ & & 1 & $3 \pm 1,4$ & & & & & & & & & & 64 & 20 & 2 & \\
\hline Ardea alba & $\begin{array}{l}\text { Garza } \\
\text { grande }\end{array}$ & $\mathrm{LC}$ & A & & $3 \pm 1,3$ & $10 \pm 0,7$ & 1 & 2 & $5 \pm 5,7$ & 3 & $1 \pm 0,6$ & & $4 \pm 2,8$ & 6 & 1 & 2 & $5 \pm 0,7$ & $2 \pm 0,7$ & 1 \\
\hline Egretta thula & Garza chica & LC & A & & 4 & $10 \pm 10,3$ & & 4 & 1 & $3 \pm 0,6$ & & & & 1 & 1 & & 1 & 6 & 3 \\
\hline $\begin{array}{l}\text { Larus } \\
\text { dominicanus }\end{array}$ & $\begin{array}{l}\text { G. } \\
\text { dominicana }\end{array}$ & $\mathrm{LC}$ & A & $10 \pm 7,1$ & & 1 & 1 & $28 \pm 19$ & $29 \pm 26,9$ & & 2 & & & & & & 3 & & \\
\hline $\begin{array}{l}\text { Chroicocephalus } \\
\text { maculipennis }\end{array}$ & $\begin{array}{l}\text { Gaviota } \\
\text { cáhuil }\end{array}$ & $\mathrm{LC}$ & A & $150 \pm 165$ & $34 \pm 32,4$ & & $83 \pm 105$ & $105 \pm 127$ & $8 \pm 9,2$ & $16 \pm 15,3$ & $6 \pm 2,1$ & $8 \pm 5,7$ & $7 \pm 6,1$ & $4 \pm 0,6$ & & $4 \pm 2,8$ & $30 \pm 28,3$ & & $4 \pm 0,7$ \\
\hline Anas flavirostris & $\begin{array}{l}\text { Pato jergón } \\
\text { chico }\end{array}$ & $\mathrm{LC}$ & A & & & & 4 & & & & & & & & & & & & \\
\hline Anas georgica & $\begin{array}{l}\text { Pato jergón } \\
\text { grande }\end{array}$ & $\mathrm{LC}$ & A & $6 \pm 6,4$ & $6 \pm 6,4$ & & $32 \pm 31,8$ & 2 & 2 & 1 & $8 \pm 8,5$ & 27 & $9 \pm 4,6$ & $6 \pm 1,7$ & $84 \pm 88,4$ & 1 & & & $23 \pm 29$ \\
\hline Anas sibilatrix & Pato real & $\mathrm{LC}$ & A & & & & 17 & & & & 3 & 20 & 5 & & $24 \pm 15,6$ & & & & 13 \\
\hline $\begin{array}{l}\text { Anas } \\
\text { cyanoptera }\end{array}$ & $\begin{array}{l}\text { Pato } \\
\text { colorado }\end{array}$ & $\mathrm{LC}$ & A & & & & & & & & 5 & 3 & & & $32 \pm 36,8$ & & & & \\
\hline $\begin{array}{l}\text { Vanellus } \\
\text { chilensis }\end{array}$ & Queltehue & $\mathrm{LC}$ & $\mathrm{A} / \mathrm{T}$ & $15 \pm 2,9$ & $13 \pm 5,3$ & $12 \pm 4,0$ & $29 \pm 13,5$ & $27 \pm 31$ & $7 \pm 3,5$ & 3 & $41 \pm 39,9$ & $13 \pm 11,3$ & $7 \pm 5,7$ & $12 \pm 0,6$ & $11 \pm 6$ & $12 \pm 2,8$ & $10 \pm 5,5$ & 2 & $8 \pm 6,1$ \\
\hline $\begin{array}{l}\text { Milvago } \\
\text { chimango }\end{array}$ & Tiuque & $\mathrm{LC}$ & $\mathrm{T}$ & & 2 & $3 \pm 2$ & 1 & & 1 & $2 \pm 1,2$ & & $4 \pm 2,8$ & 2 & $5 \pm 1,2$ & & 3 & $2 \pm 1,2$ & $2 \pm 1$ & \\
\hline $\begin{array}{l}\text { Fulica } \\
\text { armillata }\end{array}$ & $\begin{array}{l}\text { Tagua } \\
\text { común }\end{array}$ & $\mathrm{LC}$ & A & & & & & & & & & $15 \pm 13,4$ & $7 \pm 2,1$ & & $139 \pm 78$ & 1 & & & \\
\hline $\begin{array}{l}\text { Phalacrocorax } \\
\text { brasilianus }\end{array}$ & Yeco & $\mathrm{LC}$ & A & & & & & & & & & & & 1 & & & & & \\
\hline $\begin{array}{l}\text { Tachycineta } \\
\text { meyeni }\end{array}$ & $\begin{array}{l}\text { Golondrina } \\
\text { chilena }\end{array}$ & $\mathrm{LC}$ & $\mathrm{A} / \mathrm{T}$ & & 10 & 1 & & $15 \pm 0,7$ & $15 \pm 0,7$ & & & & 2 & & & 30 & & & \\
\hline $\begin{array}{l}\text { Cygnus } \\
\text { melancoryphus }\end{array}$ & $\begin{array}{l}\text { Cisne c. } \\
\text { negro }\end{array}$ & EN & A & & 2 & & & & & & & 12 & $10 \pm 2,8$ & & $22 \pm 20$ & $13 \pm 16,3$ & & & \\
\hline
\end{tabular}




\begin{tabular}{|c|c|c|c|c|c|c|c|c|c|c|c|c|c|c|c|c|c|c|c|}
\hline \multirow[t]{2}{*}{ Especie } & \multirow[t]{2}{*}{$\begin{array}{l}\text { Nombre } \\
\text { común }\end{array}$} & \multirow{2}{*}{$\begin{array}{c}\text { Estado de } \\
\text { conserva- } \\
\text { ción }\end{array}$} & \multirow{2}{*}{$\begin{array}{c}\text { Uso } \\
\text { de } \\
\text { hábitat }\end{array}$} & \multicolumn{4}{|c|}{ Isla Momberg } & \multicolumn{4}{|c|}{ Pto. Trumao La Unión } & \multicolumn{4}{|c|}{ Pto. Trumao Quilacahuin } & \multicolumn{4}{|c|}{ Currupulli } \\
\hline & & & & $\begin{array}{c}\text { Inv } \\
2017\end{array}$ & $\begin{array}{l}\text { Prim } \\
2017\end{array}$ & $\begin{array}{l}\text { Ver } \\
2018\end{array}$ & $\begin{array}{c}\text { Inv } \\
2018\end{array}$ & $\begin{array}{c}\text { Inv } \\
2017\end{array}$ & $\begin{array}{l}\text { Prim } \\
2017\end{array}$ & $\begin{array}{l}\text { Ver } \\
2018\end{array}$ & $\begin{array}{l}\text { Inv } \\
2018\end{array}$ & $\begin{array}{c}\text { Inv } \\
2017\end{array}$ & $\begin{array}{l}\text { Prim } \\
2017\end{array}$ & $\begin{array}{c}\text { Ver } \\
2018\end{array}$ & $\begin{array}{c}\text { Inv } \\
2018\end{array}$ & $\begin{array}{c}\text { Inv } \\
2017\end{array}$ & $\begin{array}{l}\text { Prim } \\
2017\end{array}$ & $\begin{array}{c}\text { Ver } \\
2018\end{array}$ & $\begin{array}{c}\text { Inv } \\
2018\end{array}$ \\
\hline $\begin{array}{l}\text { Hymenops } \\
\text { perspicillatus }\end{array}$ & Run-run & $\mathrm{LC}$ & $\mathrm{A} / \mathrm{T}$ & & $2 \pm 1,5$ & $1 \pm 0,6$ & & & 1 & 1 & & & & & & & $2 \pm 0,8$ & 1 & \\
\hline $\begin{array}{l}\text { Rollandia } \\
\text { rolland }\end{array}$ & Pimpollo & $\mathrm{LC}$ & A & $3 \pm 2,8$ & 1 & & & & & & & & & & 26 & 1 & & & \\
\hline Bubulcus ibis & $\begin{array}{l}\text { Garza } \\
\text { boyera }\end{array}$ & $\mathrm{LC}$ & $\mathrm{A} / \mathrm{T}$ & & $16 \pm 19,7$ & $11 \pm 3,6$ & & & $7 \pm 3,1$ & 3 & & & $6 \pm 3,2$ & $16 \pm 20,2$ & & & $52 \pm 62,9$ & 2 & \\
\hline Sturnella loyca & Loica & $\mathrm{LC}$ & $\mathrm{T}$ & & & & & & & & & & 1 & 38 & & & & & \\
\hline $\begin{array}{l}\text { Coragyps } \\
\text { atratus }\end{array}$ & $\begin{array}{l}\text { Jote } \mathrm{c} . \\
\text { negra }\end{array}$ & $\mathrm{LC}$ & $\mathrm{T}$ & & & 2 & & & 1 & & & & 1 & & & & & 2 & \\
\hline Ardea cocoi & Garza cuca & $\mathrm{LC}$ & A & & & 1 & & & & & & & & & 1 & & & 2 & \\
\hline $\begin{array}{l}\text { Zenaida } \\
\text { auriculata }\end{array}$ & Tórtola & $\mathrm{LC}$ & $\mathrm{T}$ & & 4 & & & & & & & & 3 & & & & & & \\
\hline Plegadis chihi & $\begin{array}{l}\text { Cuervo de } \\
\text { pantano }\end{array}$ & EN & $\mathrm{A} / \mathrm{T}$ & & & 40 & & & & & & & & & & & & & \\
\hline $\begin{array}{l}\text { Numenius } \\
\text { phaeopus }\end{array}$ & Zarapito & $\mathrm{LC}$ & A & & & & & & & & & & & & 5 & & & & \\
\hline $\begin{array}{l}\text { Enicognathus } \\
\text { leptorhynchus }\end{array}$ & Choroy & $\mathrm{LC}$ & $\mathrm{T}$ & & & & & & & & 300 & & & & & & & & \\
\hline $\begin{array}{l}\text { Coscoroba } \\
\text { coscoroba }\end{array}$ & $\begin{array}{l}\text { Cisne } \\
\text { coscoroba }\end{array}$ & EN & A & & & & & & & & & & & & 7 & & & & \\
\hline $\begin{array}{l}\text { Himantopus } \\
\text { mexicanus }\end{array}$ & Perrito & $\mathrm{LC}$ & A & & & & & & & & & & & & $8 \pm 8,5$ & & & & \\
\hline \multirow[t]{4}{*}{ Podiceps major } & Huala & $\mathrm{LC}$ & A & & & & & & & & & & & & 1 & 5 & & & \\
\hline & Riqueza & & & 6 & 14 & 13 & 9 & 8 & 12 & 9 & 9 & 9 & 14 & 10 & 15 & 12 & 10 & 10 & 7 \\
\hline & Abundancia & & & 205,1 & 106,5 & 102,5 & 192,3 & 188,5 & 81,7 & 38,1 & 372,3 & 162,5 & 66,8 & 92 & 367,6 & 140,5 & 127,4 & 26,5 & 62,3 \\
\hline & $\begin{array}{l}\text { Diversidad } \\
\mathrm{H}^{\prime}\end{array}$ & & & 0,422 & 0,935 & 0,856 & 0,689 & 0,598 & 0,86 & 0,783 & 0,34 & 0,803 & 1,065 & 0,787 & 0,832 & 0,739 & 0,723 & 0,919 & 0,715 \\
\hline
\end{tabular}


Tab. II. Comparación pareada de los índices de diversidad (H') y su varianza asociada para las distintas estaciones climáticas en los distintos puntos de monitoreo en Chile. $(*)$ Diferencias significativas $(P<0,05)$

\begin{tabular}{|c|c|c|c|c|}
\hline Isla Momberg & Invierno 2017 & Primavera 2017 & Verano 2018 & Invierno 2018 \\
\hline Invierno 2017 & - & $10,058 \pm 0,051(*)$ & $8,056 \pm 0,054(*)$ & $6,357 \pm 0,04(*)$ \\
\hline Primavera 2017 & & - & $1,386 \pm 0,057$ & $5,348 \pm 0,05(*)$ \\
\hline Verano 2018 & & & - & $3,39 \pm 0,049(*)$ \\
\hline Invierno 2018 & & & & - \\
\hline \multicolumn{5}{|l|}{ Pto. Trumao La Unión } \\
\hline Invierno 2017 & - & $4,916 \pm 0,053(*)$ & $3,014 \pm 0,072(*)$ & $3,705 \pm 0,04(*)$ \\
\hline Primavera 2017 & & - & $0,584 \pm 0,077$ & $10,22 \pm 0,05(*)$ \\
\hline Verano 2018 & & & - & $6,81 \pm 0,06(*)$ \\
\hline Invierno 2018 & & & & - \\
\hline \multicolumn{5}{|c|}{ Pto. TrumaoQuilacahuin } \\
\hline Invierno 2017 & - & $6,78 \pm 0,041(*)$ & $0,33 \pm 0,049$ & $0,808 \pm 0,036$ \\
\hline Primavera 2017 & & - & $5,74 \pm 0,05(*)$ & $6,47 \pm 0,038(*)$ \\
\hline Verano 2018 & & & - & $0,947 \pm 0,047$ \\
\hline Invierno 2018 & & & & - \\
\hline \multicolumn{5}{|l|}{ Currupulli } \\
\hline Invierno 2017 & - & $0,036 \pm 0,0547$ & $2,77 \pm 0,065(*)$ & $0,449 \pm 0,054$ \\
\hline Primavera 2017 & & - & $2,857 \pm 0,064(*)$ & $0,423 \pm 0,052$ \\
\hline Verano 2018 & & & - & $3,24 \pm 0,063(*)$ \\
\hline Invierno 2018 & & & & - \\
\hline
\end{tabular}

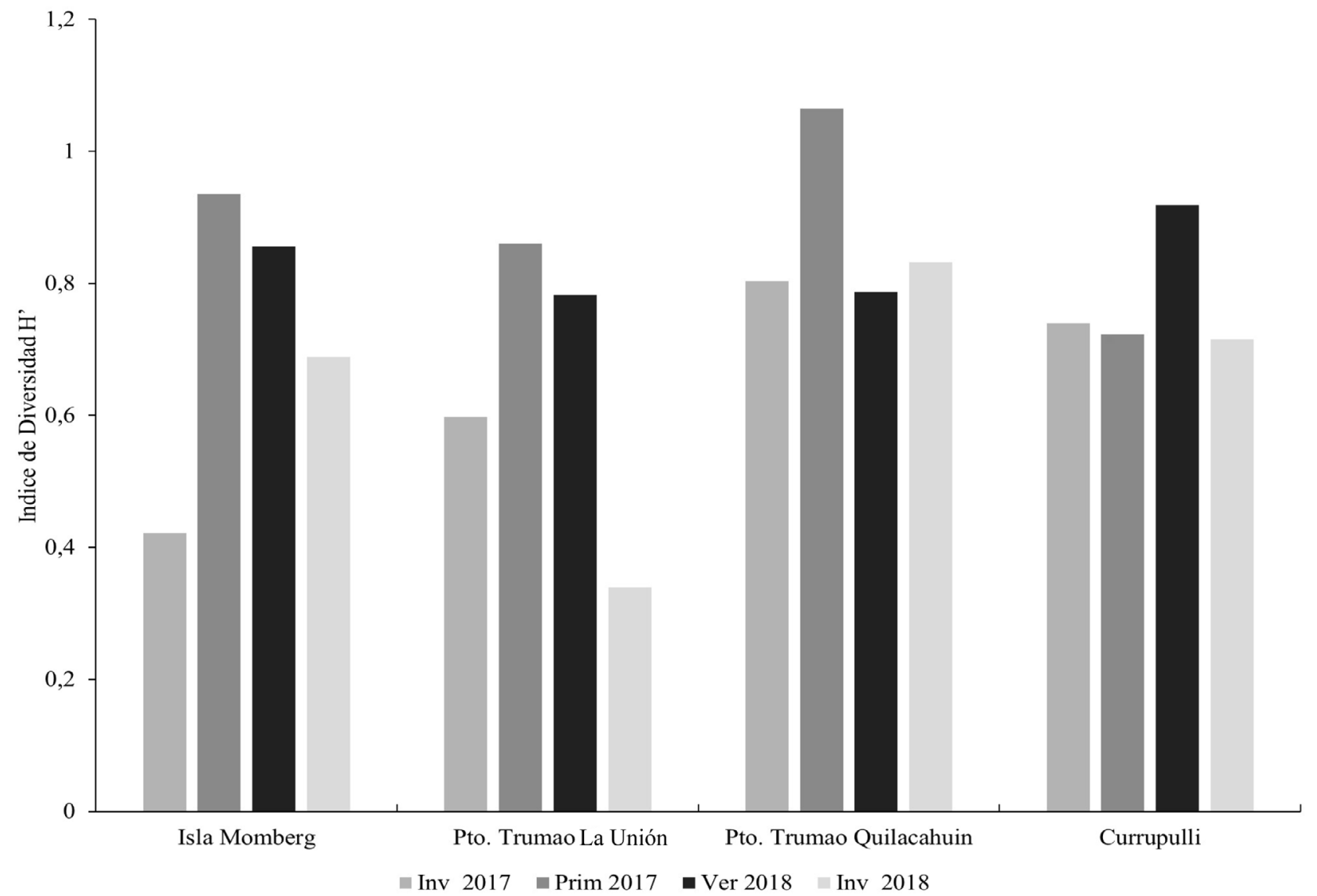

Fig. 3. Comparación estacional de la diversidad de aves en los distintos puntos de monitoreo, Chile. 


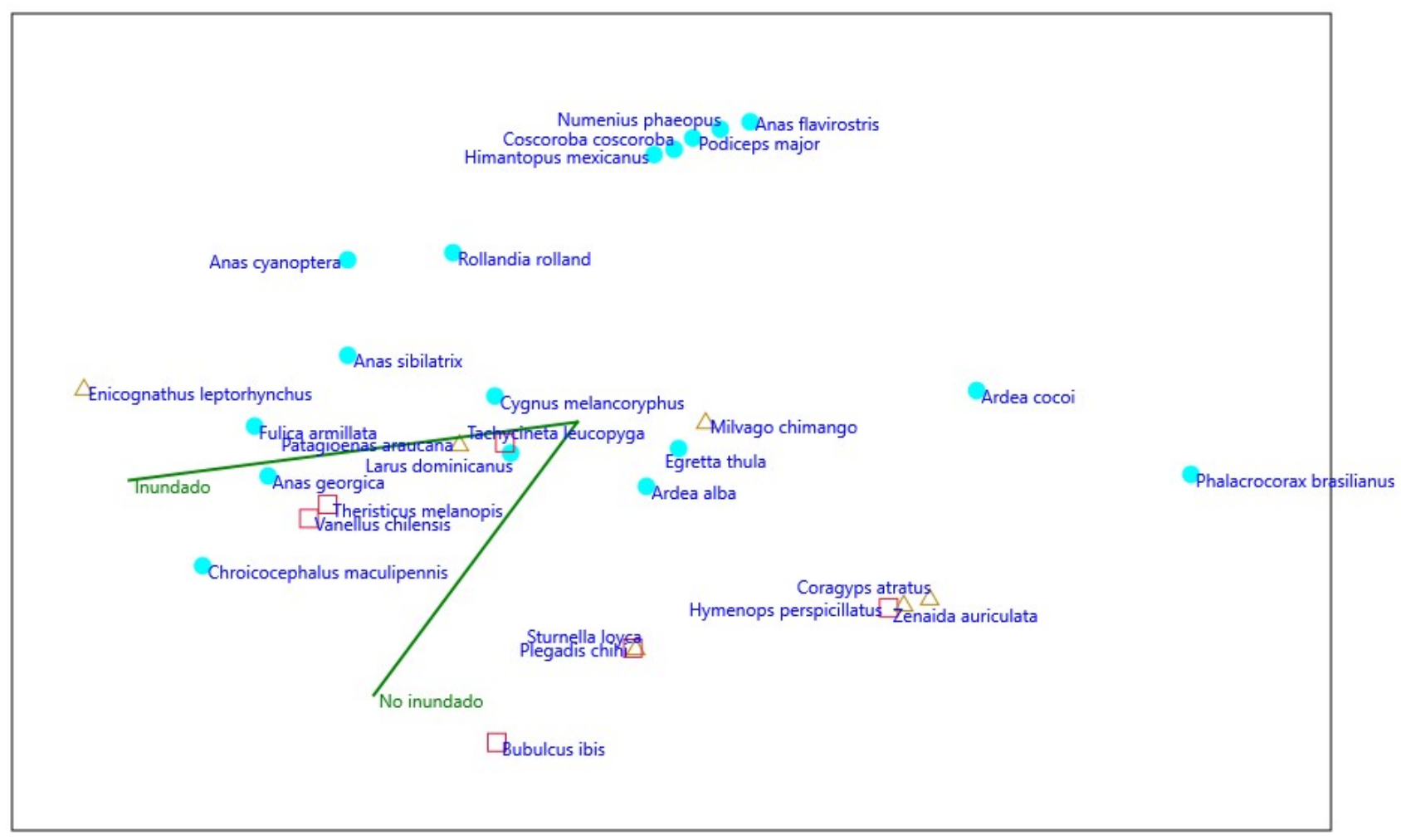

Fig. 4. Resultado del Análisis de Escalamiento Multidimensional no Metrico (MDS por su sigla en inglés) para los períodos inundados y no inundados y uso de hábitat de cada especie. Punto calipso ( $\bigcirc)$ : Acuática; Cuadrado rojo ( $\square$ ): Acuático/Terrestre y Triángulo dorado $(\triangle)$ : terrestre.

\section{DISCUSIÓN}

Los humedales fluviales son considerados como sistemas altamente dinámicos, donde las fluctuaciones hidrológicas modifican las condiciones del hábitat y, por ende, fuerzan la variabilidad temporal del ensamble de aves (LORENZÓN et al., 2019). En este estudio se observaron diferencias en la diversidad de especies entre las estaciones climáticas de invierno; inundado (invierno 2017 e invierno 2018) y las estaciones climáticas estivales; no inundado (primavera 2017 y verano 2018, Fig. 2), atribuible a los efectos de la estacionalidad climática (DGA, 2014; LORENZÓN et al., 2019). Nuestros datos concuerdan con lo reportado por LóPez de CASENAVE \& Filipello (1995) quienes registraron variaciones en la riqueza de aves entre las estaciones climáticas invernales y las estaciones climáticas estivales para la Reserva Costanera Sur, Argentina, al igual que para otros humedales neotropicales, registrando una mayor riqueza del ensamble de aves durante la época de primavera-verano (RomANo et al., 2005; IPPI et al., 2009; RonCHI-VIRGOLINI et al., 2011).

Las variaciones diarias, estacionales y anuales en la hidrología de los humedales regulan procesos ecológicos clave asociados con la diversidad biológica (KEDDY, 2002), relacionándose con la dinámica de hábitat en este tipo de ecosistemas (STANFORD et al., 2005), por lo cual, las principales diferencias en nuestro estudio se debieron a la incorporación de siete especies de aves terrestres de pastizales
(Patagioenas araucana, Milvago chimango, Sturnella loyca, Coragyps atratus, Zenaida auriculata, Enicognathus leptorhynchus) durante los periodos estivales no inundados, mientras que durante los periodos invernales inundados se incorporaron 17 especies de aves asociadas a ambientes acuáticos, siendo las más abundantes Chroicocephalus maculipennis, Anas georgica, Fulica armillata (Tab. I). Para las aves, la variación en la composición del hábitat está relacionada con la estacionalidad climática debido a que el nivel de agua influye directamente en la disponibilidad de hábitat (e.g., cambios en la composición de la vegetación, inundación) (LORENZón et al., 2019). Lo anterior, pone en evidencia que el uso de hábitat en el humedal de Trumao, por parte de las aves es altamente variable, ya que el 58,62\% prefiere el ambiente acuático y solo el $20,69 \%$ prefiere el ambiente terrestre, mientras que $20,69 \%$ ocupan ambos ambientes, este fenómeno ocurre tanto estacional como espacialmente (Fig. 4), y en donde la presencia de una u otra especie está asociada con sus particulares ciclos de vida asociados con cada ambiente en particular. Por ejemplo, la alimentación de Ardea alba (Linnaeus, 1758) (Garza grande) y Egretta thula (Molina, 1782) (Garza chica) se beneficia por el bajo nivel de las aguas que ocurre en las estaciones más secas (no inundadas), ya que se incrementa la disponibilidad de alimento, concentrando las presas en pequeñas pozas de agua de donde éstas no pueden escapar (Hafner et al., 1982; Figueroa \& Corales-Stappung, 2003). Lo anterior, es debido a la presencia de aves 
migratorias, que se encuentran en el humedal en algunas temporadas, influyen en las variaciones temporales en diversidad y en la similitud del ensamble, y que al tener las aves una alta movilidad les permite utilizar los humedales de forma oportunista, abandonado el hábitat cuando las condiciones se vuelven desfavorables y recolonizándolos cuando se vuelven favorables (CUMming et al., 2012).

Los humedales actúan como mosaicos, donde las aves raramente se encuentran distribuidas uniformemente, de esta forma, la abundancia y la composición del ensamble pueden ser influenciadas espacial y temporalmente por la heterogeneidad del área y por el número de hábitats analizados (BRANCO, 2007). En nuestro estudio, la similitud de aves entre el periodo invernal inundado y el periodo estival no inundado, obtenidas con el índice de Sorensen, nos indicó que hay similitud entre las especies registradas, sin embargo, hay diferencias significativas entre ambos periodos $(p=0,037)$; el índice de Bray-Curtis indica una disimilitud entre las abundancias de ambos periodos $(67,35 \%)$ al igual que el análisis SIMPER (79,55\%), sin embargo, la prueba U de Mann-Whitney nos indica que no hubo diferencias significativas de las abundancias entre los dos periodos $(\mathrm{p}=0,1112)$, por lo que concluimos que las abundancias se mantienen en ambos periodos de estudio, cambiando las especies que utilizan el ecosistema según el uso de hábitat (Fig. 4). Además, es importante aplicar los índices de Sorensen, Bray-Curtis y SIMPER para tener una visión más holística y así obtener mayor información sobre el ensamble de aves en los humedales.

Finalmente, estudios sobre la dinámica espacial y temporal de la fauna, especialmente de las aves, son relevantes para que las instituciones gubernamentales dispongan de la mayor información como base para la toma de decisiones sobre las mejores alternativas o usos de un área determinada (ACOSTA \& MURÚA, 2001), por lo que es fundamental seguir realizando más investigaciones en el humedal para determinar los usos (e.g., alimentación, reproducción y nidificación, etc.) que realizan las aves en los distintos hábitats y así gestionar medidas para la protección y conservación en el humedal de Trumao de manera sostenible en el tiempo.

Agradecimiento. Se agradece el apoyo oficial del proyecto interno de Investigación y Creación 2017/2018 de la Universidad Santo Tomás, "Variación estacional de aves acuáticas y mamíferos en el Humedal de Trumao, centro-sur de Chile", por el financiamiento de esta investigación.

\section{REFERENCIAS}

Acosta, J. C. \& Murúa, F. 2001. Inventario de la avifauna del Parque Natural Ischigualasto, San Juan, Argentina. Nótulas Faunísticas 3:1-9.

AcostA, J. C. \& MurúA, F. 2002. Status de conservación de la avifauna del Parque Natural Ischigualasto, San Juan, Argentina. Nótulas Faunísticas 9:1-7.

Barros, R.; Jaramillo, A. \& Schmitt, F. 2015. Lista de las aves de Chile 2014. La Chiricoca 20:79-100.

BibBy, C.; Burgess, N. \& Hill, D. 2000. Birds census technics. Cambridge, Academic Press. 139p.

Bildstein, K. L.; Bancroft, G. T.; Dugan, D. H.; Gordon, R. M.; Edwin, R. M.; Noel, E.; PAYne, L. X. \& SenNer, S. E. 1991. Approaches to the conservation of coastal wetlands in the Western Hemisphere. Wilson Bulletin 103(2):218-254.

Bodmer, R.; Mayor, P.; Antunez, M.; Chota, K.; Fang, T.; Puertas, P.; Pittet, M.; Kirkland, M.; Walkey, M.; Rios, C.; Perez-Peña, P.; Henderson, P.; Bodmer, W.; Bicerra, A.; Zegarra, J. \& Docherty, E. 2018. Major shifts in Amazon wildlife populations from recent intensification of floods and drought. Conservation Biology 32(2):333344.

Branco, J. O. 2007. Avifauna aquática do Saco da Fazenda (Itajaí, Santa Catarina, Brasil): uma década de monitoramento. Revista Brasileira de Zoología 24(4):873-882.

CANTERO, J. J. 1993. La vegetación y su relación con factores ambientales en paisajes hidrohalomórficos del centro de Argentina. Río Cuarto, Universidad Nacional de Río Cuarto.

Clarke, K. R. \& Gorley, R. N. 2006. PRIMER V6: User manual-tutorial. Plymouth Marine Laboratory.

Couve, E. \& Vidal, C. 2003. Aves de la Patagonia, Tierra del Fuego y Península Antártica, Islas Malvinas y Georgia del Sur. Punta Arenas, Fantástico Sur. 656p.

Cumming, G. S.; Paxton, M.; King, J. \& Beuster, H. 2012. Foraging guild membership explains variation in waterbird responses to the hydrological regime of an arid-region flood-pulse river in Namibia. Freshwater Biology 57(6):1202-1213.

Cursach, J. A.; Rau, J. R. \& Tobar, C. N. 2010. Aves en un humedal marino del sur de Chile. Revista de Biología Marina y Oceanografía 45(3):441-450.

DGA. 2004. Diagnóstico y clasificación de los cursos y cuerpos de agua según objetivos de calidad, cuenca del rio Bueno. Santiago, Ministerio de Obras Públicas. 147p.

Figueroa, R.; Bravo, C.; Corales, E.; LóPez, R. \& Alvarado, S. 2000. Avifauna del Santuario de la Naturaleza Los Huemules del Niblinto, región del Bío-Bío. Boletín Chileno de Ornitología 7:2-12.

FigueroA, R. A. \& CORALES-STAPPUnG, E. S. 2003. Food of Breeding Great White Egrets in an Agricultural Area of Southern Chile. Waterbirds 26(3):370-375.

Gauthier, G.; Oisgiroux, J. F.; Reed, A.; Be'Chet, A. \& Langer, L. 2005. Interactions between land use, habitat use, and population increase in greater snow geese: what are the consequences for natural wetlands? Global Change Biology 11(6):856-868.

Gallardo, J.; Rau, J. R.; DE la Fuente, A.; Marinkovic, F. \& Teutsch, C. 2018. Variación estacional de la riqueza, frecuencia relativa y diversidad de aves en humedales urbanos de Llanquihue, sur de Chile. Revista Chilena de Ornitología 24(1):27-36.

HAFNER, H.; VinCENT, B. Y. \& GoRY, G. 1982. Feeding methods, flock size and feeding success in the little egret Egretta garzetta and the squacco heron Ardeola rallides in Camargue, Southern France. Ardea 70:45-54.

Ippi, S.; Anderson, C. B.; Rozzi, R. \& ElPhick, C. S. 2009. Annual variation of abundance and composition in forest bird assemblages on navarino island, Cape Horn Biosphere Reserve, Chile. Ornitología Neotropical 20(2):231-245.

KedDY, P. A. 2002. Wetland ecology: principles and conservation. Cambridge, Cambridge University Press. 614p.

Kingsford, R. T.; Jenkins, K. M. \& Porter, J. L. 2004. Imposed hydrological stability on lakes in arid Australia and effects on waterbirds. Ecology 85(9):2478-2492. https://doi.org/10.1890/03-0470

Knutson, M. G. \& Klass, E. E. 1997. Declines in abundance and species richness of birds following a major flood on the Upper Mississippi River. Auk 114(3):367-380.

López de Casenave, J. \& Filipello, A. M. 1995. Las aves acuáticas de la Reserva Costanera Sur: cambios estacionales en la composición específica y en la abundancia de poblaciones y gremios. Hornero 14:9-14.

Lorenzón, R. E.; Beltzer, A. H.; Peltzer, P. M.; Olguín, P. F.; León, E. J.; Sovrano, L. \& RonChI-Virgolini, A. L. 2017. Habitat mediated influence of water level fluctuations on waterbird occurrence in floodplain wetlands of the Parana River, Argentina. River Research and Applications 33(9):1494-1505.

Lorenzón, R. E.; Beltzer, A. H.; Olguin, P. F.; León, H. J.; Sovrano, L. V.; Antoniazzi, C. E. \& Ronchi-Virgolini, A. L. 2019. Temporal variation of bird assemblages in dynamic fluvial wetlands: seasonality 
and influence of water level and habitat availability. Revista de Biología Tropical 67(6):1131-1145.

Martori, R.; JuÁrez, R. \& Aún, L. 2002. La taxocenosis de lagartos de Achiras, Córdova, Argentina: parámetros biológicos y estado de conservación. Revista Española de Herpetología 16:73-91.

MCALEECE, N. 1997. Biodiversity professional beta 1. Versión 1.0. The Natural History Museum and The Scottish Association for Marine Science. [on line] aVAILABLE Disponible en $<$ http://www.nhm. ac.uk/zoology/bdpro>.

MMA - Ministerio de Medio Ambiente. 2018. Plan Nacional de Protección de Humedales 2018-2022. Santiago, Ministerio de Medio Ambiente Chile. 33p.

Montoya, C. 2000. Aspectos legales vinculados a la conservación de los humedales en Chile. Gayana 64(1):1-2.

Moreno, C. E. 2001. Métodos para medir la biodiversidad. M \& TManuales y tesis SEA, vol. 1. Zaragoza, Sociedad Entomologica Aragonesa. 84p.

Navedo, J. G.; Hahn, S.; Parejo, M.; Abad-Gómez, J. M.; Gutiérrez, J. S.; Villegas, A.; SÁnchez-GuZmán, J. M. \& Masero, J. A. 2015. Unravelling trophic subsidies of agroecosystems for biodiversity conservation: food consumption and nutrient recycling by waterbirds in Mediterranean rice fields. Science of the Total Environment 511:288-297.
OnuF, C. P.; Teal, J. M. \& VAliela, I. 1977. Interactions of nutrients, plant growth, and herbivory in a mangrove ecosystem. Ecology 58:514-526.

Pohorylow, M. L.; Gatto, A. J. \& Lancelotti, J. L. 2016. Caracterización Ambiental y Estacional del Ensamble de Aves Acuáticas de la Meseta del Lago Strobel, Patagonia, Argentina. Ornitología Tropical 27:77-87.

Romano, M.; Barberis, I.; Pagano, F. \& Maidagan, J. 2005. Seasonal and interanual variation in waterbird abundance and species composition in the Melincué saline lake, Argentina. European Journal of Wildlife Research 51:1-13.

Ronchi-Virgolini, A. L.; Blake, J. G.; Lorenzón, R. E. \& Beltzer, A. H. 2011. Bird assemblages in two types of forests in the floodplain of the lower Paraná River (Argentina). Ornitología Neotropical 22:387-404.

SAG. 2015. Cartilla para cazadores. Santiago, Servicio Agrícola Ganadero. $81 \mathrm{p}$.

StanFord, J. A.; Lorang, M. S. \& Hauer, F. R. 2005. The shifting habitat mosaic of river ecosystems. Internationale Vereinigung für Theoretische und Angewandte Limnologie: Verhandlungen 29(1):123-136.

WeLLER, M. W. 1999. Wetland birds: habitat resources and conservation implications. Cambridge, Cambridge University Press. 316p.

WCS - Wildlife Conservation Society. 2019. Humedales de Chile, 40 mil reservas de vida. Santiago, Dibam. 194p.

ZAR, J. 1999. Biostatistical analysis. 4ed. Englewood Cliffs, Prentice Hall. 663p. 